CERN-TH/98-45

DFPD 98/TH/08

\title{
A smooth massless limit for supersymmetric QCD
}

\author{
Ferruccio Feruglionfe \\ Theory Division, CERN, CH-1211 Geneva 23, Switzerland
}

\begin{abstract}
We analyse in detail the behaviour of supersymmetric QCD with a number of flavours $M$ smaller than the number of colours $N$, for quark masses smaller than the dynamically generated scale $\Lambda$. In this regime, we find it useful to move from meson superfields to Nambu-Goldstone-like variables. In particular we work out the mass spectrum and the set of decay constants that specify the interactions of the lowenergy theory. We explicitly check that masses and decay constants have a consistent behaviour under decoupling and that they satisfy current algebra requirements. Finally we speculate about the massless limit. For vanishing quark masses, and only in this case, the relation between mesons and Nambu-Goldstone variables becomes singular. When analysed in terms of the Nambu-Goldstone superfields, the massless limit exhibits a spontaneous breaking of the flavour symmetry, with massless Goldstone modes embedded in an $M^{2}$-dimensional complex moduli space. The symmetry-breaking order parameter is formally infinite, but this has the only effect of turning off the interactions between the chiral superfields. The massive case, for masses smaller than $\Lambda$, can be thought of as a perturbation around the massless case, with corrections that can be systematically computed in the effective theory.
\end{abstract}

CERN-TH/98-45

DFPD 98/TH/08

February 1998

\footnotetext{
${ }^{1}$ e-mail address: feruglio@padova.infn.it

${ }^{2}$ On leave from Dipartimento di Fisica, Università di Padova, I-35131 Padua, Italy
} 


\section{Introduction}

Supersymmetric gauge theories have become an essential laboratory to analyse nonperturbative features of relativistic quantum field theories. This is due to several peculiar properties of supersymmetric theories. In these theories, the instanton calculus is much more tractable than in ordinary quantum field theories. Moreover, the holomorphic properties of the superpotential, combined with the asymptotic behaviour and the symmetry of the Wilsonian low-energy theory, often allow a determinination of the full non-perturbative superpotential, thus making a rigorous analysis of the phase structure of the theory possible. In addition, the massless spectrum of the theory is usually quite rich, and duality relations among different theories can be conjectured by matching the global anomalies. Finally, extended supersymmetric theories are even more constrained, and the full spectrum of the low-energy effective theory, including the solitonic sector, can be determined.

Among the impressive results that have been obtained so far [1], those concerning supersymmetric QCD (SQCD) with $M$ flavours and $N$ colours, $M<N$, are very intriguing. The vacuum of the theory is completely specified by the vacuum expectation values (VEVs) of the lowest component of mesons, composite chiral superfields that parametrize the flat directions of the classical scalar potential in the massless case. In the full quantum theory only the massive case seems to provide a sensible theory. In the massless limit, the scalar potential is unstable, with vacuum configurations running to infinity. Although one expects that the generalized chiral symmetry is realized in the spontaneously broken phase, it is not clear how this phase, requiring massless chiral multiplets associated to the Goldstone modes, is reached as a smooth limit starting from the massive case.

Despite the difficulties associated to the strictly massless limit, the regime of small nonvanishing masses is expected to be under control. Owing to the large meson VEVs, the theory is weakly coupled and we can identify both the full superpotential and the Kähler potential of the low-energy effective theory. Moreover, thanks to asymptotic freedom and to complementarity [2], that is the absence of a fundamental distinction between the Higgs and the confining phases in SQCD, we can reasonably guess the properties of the system. Light and weakly interacting quark supermultiplets are expected to correspond to light colour-singlet mesons characterized by weak $\sigma$-model interactions, i.e. large decay constants. In the confining picture, spectrum and interactions are also constrained by current algebra relations, that were derived long ago [3].

While the general features of the theory in this regime are known, more specific aspects have not yet been analysed, at least to the author's knowledge. For very small quark masses, the perturbative corrections to the Kähler potential are negligible and it becomes possible to isolate pure non-perturbative effects in the relevant physical quantities. It can be of interest to work out separately the mass spectrum and the interaction terms and study their exact dependence on the input parameters. It is also interesting to see how the general current algebra requirements are implemented. Moreover, the explicit form 
of the low-energy effective Lagrangian may also shed some light on the singular massless case. The aim of the present work is to examine in detail the behaviour of the system for quark masses much smaller than the characteristic scale $\Lambda$ of the theory. To keep the description of SQCD as close as possible to the low-energy limit of ordinary QCD, we find it convenient to move from meson to Nambu-Goldstone superfields. For small quark masses the mesons fluctuate around large VEVs, while the Nambu-Goldstone variables remain close to the origin of field space. From the low-energy effective Lagrangian we can read the mass spectrum and we can naturally characterize the interactions in terms of a set of decay constants. The decay constants increase when the quark masses decrease, in agreement with the expectation that the interactions between the relevant low-energy degrees of freedom become weaker for smaller quark masses. The explicit expressions of masses and decay constants satisfy the properties required by decoupling and by current algebra.

In the last part of this note we speculate about the massless limit of the theory. This is a delicate point. As long as the quark masses are non-vanishing, meson and NambuGoldstone superfields are related by an analytic transformation. The choice of variables is irrelevant, and any physical observable remains invariant under the field redefinition. In the massless limit, the mapping between the two sets of variables become singular. It is well-known that the mesons possess run-away vacua in the massless limit [4] and the theory has at most a cosmological interpretation [5]. The metric of the scalar manifold is also singular for meson VEVs running at infinity. On the contrary, when the Nambu-Goldstone variables are used, the metric remains regular and the singular behaviour of the massless case can be restricted to a single parameter, which controls the meson decay constants. The elementary excitations of the system become massless and non-interacting in the massless limit. The scalar potential becomes completely flat. The chiral symmetry is in the spontaneously broken phase, with the expected number of chiral massless supermultiplets. Even if formally the chiral symmetry is broken by an infinite amount, the limit is reached smoothly in any physical computable quantity. For masses smaller than the SQCD scale $\Lambda$, the massive case can be seen as a small perturbation around the massless limit, as in the case of ordinary QCD.

Although we have not yet found a clear-cut test to prefer one of the two sets of variables, the present analysis suggests that a smooth massless limit of SQCD, $M<N$, is not inconceivable.

To discuss these points we proceed by recalling the notation and the well-known results for SQCD, in the $M \leq N$ case. Then we will illustrate the key points in the simplest $M=1$ case. The central part of the paper is devoted to the discussion of the general case. Then we will come to the discussion of the massless limit and finally we will conclude by summarizing the main results. 


\section{Supersymmetric QCD}

The theory is defined by the Lagrangian:

$$
\begin{aligned}
\mathcal{L}=\mathcal{L}_{S Y M} & +\int d^{4} \theta\left[\left(Q_{i}^{\alpha}\right)^{\dagger}\left(e^{V}\right)_{\alpha}^{\beta} Q_{\beta}^{i}+\tilde{Q}_{i}^{\alpha}\left(e^{-V}\right)_{\alpha}^{\beta}\left(\tilde{Q}_{\beta}^{i}\right)^{\dagger}\right] \\
& -\left[\int d^{2} \theta \hat{m}_{j}^{i} Q_{\alpha}^{i} \tilde{Q}_{j}^{\alpha}+\text { h.c. }\right]
\end{aligned}
$$

where $Q_{\alpha}^{i}$ and $\tilde{Q}_{i}^{\alpha}(\alpha=1, \ldots, N ; i=1, \ldots, M)$ are chiral superfields transforming in the $N$ and $\bar{N}$ representations of the gauge group $S U(N)$, respectively; $V$ is a set of vector superfields, suitably contracted with the $S U(N)$ generators in the fundamental representation. The first term, $\mathcal{L}_{S Y M}$, is the Lagrangian for the pure super-Yang-Mills theory. The superpotential consists just of a mass term described by the mass matrix $\hat{m}$.

At the classical level and in the massless case, $\hat{m}=0$, the vacua are distributed along flat directions, defining a classical moduli space [6]. For $M<N$ the classical moduli space can be parametrized in terms of VEVs of the lowest components of $M^{2}$ meson superfields:

$$
\mathcal{M}_{j}^{i}=Q_{\alpha}^{i} \tilde{Q}_{j}^{\alpha}
$$

In the $M=N$ case the complex moduli space is $\left(N^{2}+1\right)$-dimensional, the extra coordinate being provided by baryon composite superfields:

$$
\begin{array}{ll}
\mathcal{B}=\epsilon^{\alpha_{1} \ldots \alpha_{N}} & Q_{\alpha_{1}}^{1} \ldots Q_{\alpha_{N}}^{N} \\
\tilde{\mathcal{B}}=\epsilon_{\alpha_{1} \ldots \alpha_{N}} & \tilde{Q}_{1}^{\alpha_{1}} \ldots \tilde{Q}_{N}^{\alpha_{N}}
\end{array}
$$

subjected to the classical constraint:

$$
\operatorname{det} \mathcal{M}-\mathcal{B \mathcal { B }}=0
$$

At the generic point of the classical moduli space the $S U(N)$ gauge symmetry is spontaneously broken down to $S U(N-M)$ for $M<N-1$, and completely for $M=N-1$ and $M=N$. In the massless case the theory exhibits a global flavour symmetry under the group $S U(M) \times S U(M) \times U(1)_{B} \times U(1)_{R}$. The first three factors correspond to the usual chiral symmetry and to the baryon number, already present in the non-supersymmetric theory. The additional $U(1)_{R}$, peculiar to the supersymmetric case, is the anomaly-free combination of the axial $U(1)$ symmetry and a global continuous R-symmetry. The $U(1)_{R}$ charges of squarks, quarks and gauginos are proportional to $M-N,-N$ and $M$, respectively.

When quantum corrections are included, in the massive case $\hat{m} \neq 0$, a number of exact results can be derived. First of all the scalar potential of the theory vanishes at the minima and supersymmetry is unbroken. Moreover the $\operatorname{VEVs}\left\langle\mathcal{M}_{j}^{i}\right\rangle$ of the lowest components of the meson superfields can be determined exactly:

$$
\left\langle\mathcal{M}_{j}^{i}\right\rangle=\Lambda^{3}\left[\operatorname{det}\left(\frac{\hat{m}}{\Lambda}\right)\right]^{\frac{1}{N}}\left(\hat{m}^{-1}\right)_{j}^{i} \quad(M \leq N)
$$


where $\Lambda$ denotes the renormalization group invariant, dynamically generated scale of the theory. This result was first obtained from the Taylor-Veneziano-Yankielowicz (TVY) effective Lagrangian [4], based on the superpotential:

$$
w_{T V Y}=\mathcal{S}\left[\log \left(\frac{\mathcal{S}^{N-M} \operatorname{det} \mathcal{M}}{\Lambda^{3 N-M}}\right)-(N-M)\right]-\operatorname{tr}(\hat{m} \mathcal{M}),
$$

$\mathcal{S}$ denoting a chiral superfield describing massive degrees of freedom associated to the pure glue sector of the theory. We recall that also the Affleck-Dine-Seiberg (ADS) superpotential [7]

$$
w_{A D S}=(N-M)\left(\frac{\Lambda^{3 N-M}}{\operatorname{det} \mathcal{M}}\right)^{\frac{1}{N-M}}+\operatorname{tr}(\hat{m} \mathcal{M})
$$

gives rise, for $M<N$, to the VEVs of eq. (5). The ADF superpotential contains all nonperturbative corrections to the classical superpotential and can be related to the TVY superpotential by integrating out the chiral superfield $\mathcal{S}$ from the TVY Lagrangian. The VEVs $\left\langle\mathcal{M}_{j}^{i}\right\rangle$ have also been reproduced via explicit instanton computations [《].

The massless limit can be carried out without problems for $M=N$. In this case the quantum moduli space along the mesonic branch has complex dimension $N^{2}-1$, as a consequence of the quantum constraint [9]:

$$
\operatorname{det}\langle\mathcal{M}\rangle=\Lambda^{2 N} \quad .
$$

This reflects the spontaneous breaking of the global symmetry down to the diagonal subgroup $S U(M) \times U(1)_{B} \times U(1)_{R}$. Notice that the subgroup $U(1)_{R}$ is unbroken and each complex direction in the moduli space contains precisely 1 Goldstone mode.

The meson VEVs of eq. (5) are at the origin of the difficulty in dealing with the massless limit for $M<N$. In such a limit, divergent VEVs are obtained for $\mathcal{M}$, signalling an instability of the scalar potential of the theory. By adopting the confining picture, where the low-energy spectrum is described by gauge invariant composite operators, a powerful constraint is offered by a variant of the Dashen formula [3], relating pseudoGoldstone decay constants $F_{i}^{a}$ and masses $m_{i j}^{\pi}$ to the VEV of a double commutator

$$
F_{i}^{a} m_{i j}^{\pi} F_{j}^{b}=\left\langle 0\left|\left[Q_{5}^{a},\left[Q_{5}^{b},\left.w\right|_{\theta=0}\right]\right]\right| 0\right\rangle .
$$

The charges $Q_{5}^{a}$ denote the $S U(M) \times S U(M)$ generators that are spontaneously broken in the chiral limit and $\left.w\right|_{\theta=0}$ is the lowest-order component of the superpotential. A similar formula holds also for the $R$ charge and the related decay constant. For equal quark masses, the right-hand side of eq. (9) is proportional to the product of the quark mass times the trace of the squark condensate of eq. (5). If $m_{i j}^{\pi}$ are linear in the quark masses, as suggested by complementarity, then the above relation shows that the decay constants should diverge, in the limit of vanishing quark masses, as the square root of the meson VEVs.

All the material collected in this section is standard and well-known and we have reported it here just to set the notation and to make the presentation self-contained. 


\section{$3 \quad M=1$}

In this section we consider in detail the case $M=1$. The global symmetry is $U(1)_{B} \times U(1)_{R}$. There is a single meson $\mathcal{M}$ and the mass matrix $\hat{m}$ reduces to a single mass $m$ that, without losing generality, can be taken real and non-negative. The global symmetry is spontaneously broken down to $U(1)_{B}$ by the meson VEV. The $U(1)_{R}$ symmetry is also explicitly broken by the mass term and, for $m \neq 0$, one expects a massive chiral multiplet in the spectrum. Here we are interested in the behaviour of the theory for a small mass and we assume that $m$ is much smaller than the dynamically generated scale $\Lambda$. From eq. (5) we can read the meson VEV 円:

$$
\langle\mathcal{M}\rangle=\Lambda^{2}\left(\frac{\Lambda}{m}\right)^{\frac{N-1}{N}} .
$$

We now proceed to redefine the meson superfield according to t?:

$$
\mathcal{M}=\Lambda^{2}\left(\frac{\Lambda}{m}\right)^{\frac{N-1}{N}} \exp \left(i \xi^{0}\right) \quad .
$$

in such a way that the chiral superfield $\xi^{0}$, not yet conveniently normalized, has a vanishing VEV. The lowest component of this superfield contains the candidate Nambu-Goldstone boson for the spontaneous breaking of the global $U(1)_{R}$, as can be seen by the fact that under a $U(1)_{R}$ transformation it shifts by an amount proportional to the parameter of the transformation. To provide the correct normalization to the superfield $\xi^{0}$ we should consider the Kähler potential of the theory in the low-energy regime. By expressing it in terms of the superfield $\xi^{0}$ and by asking for a canonical kinetic term, we would be able to normalize $\xi^{0}$ appropriately.

For very large values of the meson VEVs, the original gauge theory is in the weakly coupled regime and the Kähler potential can be approximated by the classical one [5, projected along the direction of the meson superfields [13, 14:

$$
K=2 \sqrt{\mathcal{M}^{\dagger} \mathcal{M}}
$$

In terms of the superfield $\xi^{0}$, we obtain:

$$
K=2|\Lambda|^{2}\left(\frac{|\Lambda|}{m}\right)^{\frac{N-1}{N}}\left|\exp \left(i \frac{\xi^{0}}{2}\right)\right|^{2} \quad .
$$

By expanding $K$ in powers of $\xi^{0}$, we immediately see that, in order to have a canonically normalized kinetic term, the chiral superfield $\xi^{0}$ should be rescaled according to

$$
\xi^{0} \rightarrow \frac{\sqrt{2} \xi^{0}}{F}, \quad F=\Lambda\left(\frac{\Lambda}{m}\right)^{\frac{N-1}{2 N}} .
$$

\footnotetext{
${ }^{1}$ From now on, we focus on one of the $N$ independent vacua.

${ }^{2}$ Variables similar to those considered here have already been used in the literature to discuss the massless limit of SQCD [10] and to analyse the non-perturbative properties of softly broken SQCD [1].

${ }^{3}$ I thank L. Randall and R. Rattazzi for clarifying this point to me. Logarithmic corrections to the Kähler potential [12] become negligible for $m \ll \Lambda$.
} 
The meson superfield now reads:

$$
\mathcal{M}=F^{2} \exp \left(i \frac{\sqrt{2} \xi^{0}}{F}\right) \quad .
$$

The full low-energy effective Lagrangian is defined by the Kähler potential:

$$
K=2|F|^{2}\left|\exp \left(i \frac{\xi^{0}}{\sqrt{2} F}\right)\right|^{2} \quad
$$

and by the ADS superpotential, which takes the form:

$$
w=m F^{2}\left[(N-1) \exp \left(-i \frac{\sqrt{2}}{N-1} \frac{\xi^{0}}{F}\right)+\exp \left(i \frac{\sqrt{2} \xi^{0}}{F}\right)\right] \quad .
$$

The constant $F$ has clearly the meaning of the decay constant associated to the lowest component of $\xi^{0}$. When we consider a very small mass $m \ll \Lambda, F$ is much larger than the SQCD scale $\Lambda$. If we are interested in field fluctuations and energy scales smaller than or comparable to $F$, we can expand the exponentials in eqs. (16) and (17) in powers of $\xi^{0} / F$, obtaining:

$$
\begin{gathered}
K=\left|\xi^{0}\right|^{2}+\frac{i}{2 \sqrt{2}}\left|\xi^{0}\right|^{2}\left(\frac{\xi^{0}}{F}-\frac{\bar{\xi}^{0}}{\bar{F}}\right)+\ldots \\
w=m\left(\frac{N}{N-1}\left(\xi^{0}\right)^{2}-\frac{\sqrt{2} i}{3} \frac{N(N-2)}{(N-1)^{2}} \frac{\left(\xi^{0}\right)^{3}}{F}+\ldots\right)
\end{gathered}
$$

Dots in the expression of $K$ denote analytic or anti-analytic terms and higher-order terms in the expansion, whereas in the expression of $w$ they stand for an unessential constant and higher-order contributions. The chiral superfield $\xi^{0}$ describes two neutral spin-0 particles and a Majorana particle of spin 1/2. They have a common mass, given by:

$$
m_{\xi^{0}}=\frac{2 N}{N-1} m
$$

This mass is larger than twice the original quark mass. It approaches $2 m$ in the large- $N$ limit. Notice the difference with ordinary QCD. In that case, the squared pseudoscalar masses scale linearly with the quark current mass and, for light flavours, there is a substantial contribution from the quark-antiquark condensate. In the supersymmetric case, it is the mass of the supermultiplet $\xi^{0}$ that scales linearly with the quark mass, and there is no contribution from the quark-antiquark condensate.

As recalled above, $m_{\xi^{0}}$ and $F$ should satisfy a supersymmetric variant of the Dashen formula, which can, for $M=1$, be cast in the following form:

$$
m_{\xi^{0}} F^{2}=\left\langle 0\left|\left[Q_{R},\left[Q_{R},\left.w\right|_{\theta=0}\right]\right]\right| 0\right\rangle \quad .
$$

Both the $R$ charge $Q_{R}$ and the decay constant $F$ refers to a convenient normalization of the $R$-current and its relevant matrix element. Under a $U(1)_{R}$ transformation, the lowest component of $\xi^{0}$ shifts by an amount proportional to $F$. We see that the right-hand side coincides with the double derivative of $w$, evaluated at $\xi^{0}=0$, times $F^{2}$, in agreement with the left-hand side. 


\section{$4 \quad 1<M<N$}

In this section we generalize the considerations of the previous section to $M$ smaller than $N$ but otherwise arbitrary. It is not restrictive to consider a diagonal mass matrix $\hat{m}$ with non-negative entries $m_{1}, \ldots, m_{M}$.

The first step consists in performing an analytic transformation from the meson variables $\mathcal{M}_{j}^{i}$ to $\mathrm{Nambu}-$ Goldstone-like variables. In a matrix notation:

$$
\mathcal{M}=\Lambda^{2}\left[\frac{\Lambda}{(\operatorname{det} \hat{m})^{\frac{1}{M}}}\right]^{\frac{N-M}{N}}(\operatorname{det} \hat{m})^{\frac{1}{M}} \hat{m}^{-1} \exp \left(i \xi^{a} T^{a}\right)
$$

We have $M^{2}$ chiral superfields $\xi^{a}\left(a=0, \ldots, M^{2}-1\right)$. The $M \times M$ matrices $T^{a}$ denote the generators of $U(M)$ in the fundamental representation, normalized for convenience

according to $\operatorname{tr}\left(T^{a} T^{b}\right)=2 \delta^{a b}$. We identify with $T^{0}$ the generator equal to $\sqrt{2 / M}$ times the unit matrix.

In terms of the new variables, the VEVs of eq. (5) read:

$$
\left\langle\xi^{0}\right\rangle=\left\langle\xi^{1}\right\rangle=\ldots=\left\langle\xi^{M^{2}-1}\right\rangle=0 \quad .
$$

The ADS superpotential, expressed in terms of the new variables, becomes:

$$
w=\Lambda^{2}\left[\frac{\Lambda}{(\operatorname{det} \hat{m})^{\frac{1}{M}}}\right]^{\frac{N-M}{N}}(\operatorname{det} \hat{m})^{\frac{1}{M}}\left[(N-M) \exp \left(i-\frac{\sqrt{2 M}}{N-M} \xi^{0}\right)+\operatorname{tr}\left(\exp \left(i \xi^{a} T^{a}\right)\right)\right]
$$

To provide canonical kinetic terms, the Nambu-Goldstone chiral superfields should be conveniently rescaled. This can be done by analysing the Kähler potential of the theory, expressed in terms of the Nambu-Goldstone supermultiplets. For small field and energy variations around the large meson VEV, the full Kähler potential can be approximated by the classical one:

$$
\begin{aligned}
K & =2 \operatorname{tr} \sqrt{\mathcal{M}^{\dagger} \mathcal{M}} \\
& =2|\Lambda|^{2}\left[\frac{|\Lambda|}{(\operatorname{det} \hat{m})^{\frac{1}{M}}}\right]^{\frac{N-M}{N}} \operatorname{tr}\left[\exp \left(-i \bar{\xi}^{a} T^{a}\right)(\operatorname{det} \hat{m})^{\frac{2}{M}} \hat{m}^{-2} \exp \left(i \xi^{a} T^{a}\right)\right]^{\frac{1}{2}}
\end{aligned}
$$

The Kähler potential $K$ and the superpotential $w$ would allow a full analysis of the lowenergy theory. Nevertheless, instead of dealing with the general case, it is more instructive to work out two particular examples, which we believe to be sufficiently representative of the behaviour of the system.

\section{$4.1 \hat{m}=\operatorname{diag}(m, \ldots, m)$}

The first example is characterized by a generic $M<N$, with a degenerate mass matrix $\hat{m}=\operatorname{diag}(m, \ldots, m)$. In this case the Kähler potential of eq. (25) specializes to:

$$
K=2|\Lambda|^{2}\left(\frac{|\Lambda|}{m}\right)^{\frac{N-M}{N}} \operatorname{tr}\left[\exp \left(-i \bar{\xi}^{a} T^{a}\right) \exp \left(i \xi^{a} T^{a}\right)\right]^{\frac{1}{2}}
$$


Because of the degeneracy of the quark mass matrix, the required rescaling of the NambuGoldstone superfields is uniform:

$$
\xi^{a} \rightarrow \frac{\xi^{a}}{F}, \quad F=\Lambda\left(\frac{\Lambda}{m}\right)^{\frac{N-M}{2 N}} .
$$

The relation between meson and Nambu-Goldstone superfields is:

$$
\mathcal{M}=F^{2} \exp \left(i \frac{\xi^{a} T^{a}}{F}\right)
$$

The theory is described by the Kähler potential:

$$
\begin{aligned}
K & =2|F|^{2} \operatorname{tr}\left[\exp \left(-i \frac{\bar{\xi}^{a} T^{a}}{\bar{F}}\right) \exp \left(i \frac{\xi^{a} T^{a}}{F}\right)\right]^{\frac{1}{2}} \\
& =\left|\xi^{a}\right|^{2}+\ldots
\end{aligned}
$$

and by the superpotential:

$$
\begin{aligned}
w & =m F^{2}\left[(N-M) \exp \left(-i \frac{\sqrt{2 M}}{N-M} \frac{\xi^{0}}{F}\right)+\operatorname{tr}\left(\exp \left(i \frac{\xi^{a} T^{a}}{F}\right)\right)\right] \\
& =-m\left[\left(\frac{N}{N-M}\right)\left(\xi^{0}\right)^{2}+\left(\xi^{1}\right)^{2}+\ldots+\left(\xi^{M^{2}-1}\right)^{2}+\ldots\right]
\end{aligned}
$$

In the above expressions we have also listed the first relevant terms in the expansion in powers of $\xi^{a} / F$. All the interaction terms are down by powers of $1 / F$ compared to the quadratic part. The spectrum consists of one chiral supermultiplet with mass

$$
m_{\xi^{0}}=\frac{2 N}{N-M} m
$$

and $M^{2}-1$ degenerate supermultiplets with masses

$$
m_{\xi^{1}}=\ldots=m_{\xi^{M^{2}-1}}=2 m
$$

Concerning the scaling of the masses with $m$, the same remarks as made in the previous section apply. Notice that $M^{2}-1$ superfields have a common mass that is exactly twice the quark mass. The mass of the remaining superfield approaches this value in the large- $N$ limit. As a curiosity, we note that, although this analysis does not cover the case $M=N$, if we naively try to extrapolate the spectrum to that case, we see that the mass of the singlet diverges, in qualitative agreement with the fact that, for $M=N$, only $N^{2}-1$ chiral superfields remain massless along the mesonic branch.

It can easily be checked that also in this case the Dashen-Veneziano relation, eq. (9), is satisfied. Finally, notice that in the large- $N$ limit the anomaly-free $U(1)_{R}$ symmetry tends to the axial $U(1)_{A}$ and the global flavour symmetry is promoted to $U(M) \times U(M)$. In the case under discussion, this symmetry is broken by the mass matrix down to the diagonal $U(M)$. We expect $M^{2}$ degenerate pseudoGoldstone multiplets, as indeed can be seen by sending $N$ to infinity in eq. (31). 


\section{$4.2 \quad M=2$}

As a further example we consider in detail the case $M=2$, for a general mass matrix $\hat{m}=\operatorname{diag}\left(m_{1}, m_{2}\right)$. After some algebra, the quadratic part of the Kähler potential given in eq. (25), and specialized to $M=2$, reads:

$$
\begin{aligned}
K & =|\Lambda|^{2}\left(\frac{|\Lambda|}{\sqrt{m_{1} m_{2}}}\right)^{\frac{N-2}{N}}\left[\sqrt{\frac{m_{1}}{m_{2}}}\left|\frac{\xi^{0}+\xi^{3}}{\sqrt{2}}\right|^{2}+\sqrt{\frac{m_{2}}{m_{1}}}\left|\frac{\xi^{0}-\xi^{3}}{\sqrt{2}}\right|^{2}\right. \\
& \left.+\frac{2 m_{1}}{m_{1}+m_{2}} \sqrt{\frac{m_{1}}{m_{2}}}\left|\frac{\xi^{1}+i \xi^{2}}{\sqrt{2}}\right|^{2}+\frac{2 m_{2}}{m_{1}+m_{2}} \sqrt{\frac{m_{2}}{m_{1}}}\left|\frac{\xi^{1}-i \xi^{2}}{\sqrt{2}}\right|^{2}+\ldots\right] .
\end{aligned}
$$

As before, dots denote purely analytic or anti-analytic terms. To recover canonical kinetic terms, a common rescaling of the fields is no longer sufficient, and several decay constants will appear, as expected, since the masses are not degenerate. We first rotate the NambuGoldstone fields to the natural combinations:

$$
\eta^{ \pm}=\frac{\xi^{0} \pm \xi^{3}}{\sqrt{2}}, \quad \xi^{ \pm}=\frac{\xi^{1} \pm i \xi^{2}}{\sqrt{2}}
$$

Then we perform the rescaling:

$$
\begin{array}{rlrl}
\eta^{+} \rightarrow \frac{\eta^{+}}{F_{\eta^{+}}}, & F_{\eta^{+}} & =\Lambda\left[\frac{\Lambda}{m_{1}^{-\frac{1}{N-2}} m_{2}^{\frac{N-1}{N-2}}}\right]^{\frac{N-2}{2 N}} \\
\eta^{-} \rightarrow \frac{\eta^{-}}{F_{\eta^{-}}}, & F_{\eta^{-}} & =\Lambda\left[\frac{\Lambda}{m_{1}^{\frac{N-1}{N-2}} m_{2}^{-\frac{1}{N-2}}}\right]^{\frac{N-2}{2 N}} \\
\xi^{+} \rightarrow \frac{\xi^{+}}{F_{\xi^{+}}}, & F_{\xi^{+}}=\Lambda\left[\frac{\Lambda}{m_{1}^{-\frac{N+1}{N-2}} m_{2}^{\frac{N-1}{N-2}}\left(\frac{m_{1}+m_{2}}{2}\right)^{\frac{N}{N-2}}}\right]^{\frac{N-2}{2 N}} \\
\xi^{-} \rightarrow \frac{\xi^{-}}{F_{\xi^{-}}}, & F_{\xi^{-}}=\Lambda\left[\frac{\Lambda}{m_{1}^{\frac{N-1}{N-2}} m_{2}^{-\frac{N+1}{N-2}}\left(\frac{m_{1}+m_{2}}{2}\right)^{\frac{N}{N-2}}}\right]^{\frac{N-2}{2 N}}
\end{array}
$$

The kinetic terms of the rescaled variables are canonical. The quadratic part of the superpotential is given by:

$$
\begin{aligned}
w= & -\left[\left(m_{1}+m_{2}\right) \xi^{+} \xi^{-}+\right. \\
& \left.\left(\frac{N-1}{N-2}\right) m_{2}\left(\eta^{+}\right)^{2}+\left(\frac{N-1}{N-2}\right) m_{1}\left(\eta^{-}\right)^{2}+\left(\frac{2}{N-2}\right) \sqrt{m_{1} m_{2}} \eta^{+} \eta^{-}\right]
\end{aligned}
$$

The masses of the physical states can be readily obtained from this expression. As a first

check, when $m_{1}=m_{2}=m$, we can verify that the spectrum consists of three degenerate supermultiplets of mass $2 m$ plus a supermultiplet of mass $2 m N /(N-2)$, as required by 
eqs. (31) and (32). Also the four decay constants become equal and reproduce the value of eq. (27).

An important, additional check of the present picture is provided by the limit $m_{1} \ll$ $m_{2}$ f. In this case, at energies much smaller than $m_{2}$, the theory should look like SQCD with a single flavour. Indeed, in this limit, three of the four chiral supermultiplets have masses growing with $m_{2}$, whereas the last supermultiplet, essentially given by $\eta^{-}$, has a mass $2 m_{1} N / N-1$, as can be checked from eq. (36), by including the first-order correction to the lowest-order eigenvalue. Moreover, the decay constant $F_{\eta^{-}}$of the light supermultiplet exactly reproduces that of the $M=1$ case. To appreciate this fact, we should remember that the renormalization group-invariant scales of the theories with one and two flavours are related by:

$$
\Lambda_{(1)}^{3 N-1}=\Lambda^{3 N-2} m_{2}
$$

when the matching between the two theories is made at the scale $m_{2}$. Taking into account this relation, the decay constant $F_{\eta^{-}}$can be expressed as

$$
F_{\eta^{-}}=\Lambda_{(1)}\left(\frac{\Lambda_{(1)}}{m_{1}}\right)^{\frac{N-1}{N}}
$$

exactly as required by the $M=1$ case. We stress that this fundamental property does not hold for an arbitrary choice of the Kähler potential $K$. This was probably at the origin of the early difficulties in discussing the regime of small quark masses.

\section{The massless limit}

At this point it is appropriate to make some comment on the massless case. In ordinary QCD, the massless limit is smooth. The flavour symmetry is spontaneously broken and massless Nambu-Goldstone bosons appear. The decay constant of the pseudoscalar mesons is of the order of the QCD scale. When small non-vanishing quark masses are turned on, the theory can be regarded as a small perturbation around the massless case and corrections can be systematically included order by order in the symmetry-breaking parameters. In SQCD, $M<N$, the lowest components of the meson superfields have a scalar potential with run-away vacua in the massless limit. This seems to prevent from the very beginning the construction of the quantum theory. The fate of the global symmetries in the massless limit then becomes an ill-defined question. Apparently, the massive case can no longer be regarded as a perturbation around the massless limit.

We may expect to detect some signals associated to this singular limit already when the quark masses are non-vanishing but very small. What emerges, instead, from the present analysis, is that the massless limit, whether it exists or not as a quantum theory, is approached smoothly. We recall that throughout this analysis we have made use of the

\footnotetext{
${ }^{4}$ Both masses are kept not larger than $\Lambda$.
} 
Nambu-Goldstone variables, introduced with the purpose of being as close as possible to the standard description of low-energy QCD. For any non-vanishing value of $m$ the NambuGoldstone variables are related to the meson variables by an analytic transformation, explicitly given in eqs. (15), (22) and (28). While the Nambu-Goldstone superfields are perhaps convenient and have a direct physical interpretation, their use is not at all compelling, and we could have derived exactly the same mass spectrum and the same interactions by working in terms of meson variables. Indeed, for $m \neq 0$, the choice between the two sets of variables is completely irrelevant and the physical properties of the system are exactly the same, as expected on the basis of general results on quantum field theory. The relation between the two sets of superfields is, however, singular at $m=0$ and the two descriptions are no longer equivalent.

Consider, for instance, the case $M=1$. By going back to the results of section 3, we see that in the limit $m \rightarrow 0$ the supermultiplet $\xi^{0}$ becomes massless. Indeed, if a smooth massless limit exists, we expect precisely one massless scalar signalling the spontaneous breakdown of the global $U(1)_{R}$. The other states should then be degenerate with the Nambu-Goldstone mode because supersymmetry is unbroken. The presence of a massless supermultiplet is confirmed by an inspection of the scalar potential of the theory

$$
V=2 m^{2}|F|^{2}\left|\exp \left(i \frac{\xi^{0}}{\sqrt{2} F}\right)-\exp \left(-i \frac{N+1}{N-1} \frac{\xi^{0}}{\sqrt{2} F}\right)\right|^{2}
$$

For any non-vanishing value of $m, V$ reaches its minimum at $\xi^{0}=0$. When $m$ goes to zero, $V$ vanishes identically, leading to unbroken supersymmetry and to a complex flat direction.

Notice that the metric of the scalar manifold is given by $1 /\left(2 \sqrt{\mathcal{M M}^{\dagger}}\right)$ in terms of mesons and becomes singular at the minimum of the scalar potential, when $m$ tends to zero. Such a singularity is however absent when we look at quantities that are invariant under reparametrizations of the fields 1 . For example, the curvature of the scalar manifold vanishes at any value of $\mathcal{M}$. At the same time, the metric associated to $\xi^{0}$, $\left|\exp \left(i \xi^{0} / \sqrt{2} F\right)\right|^{2}$, is regular in the vicinity of $\xi^{0}=0$, for any $m \ll \Lambda$.

We also see that in the $m \rightarrow 0$ limit, with $\Lambda$ fixed, the decay constant $F$ diverges, contrary to what happens in ordinary QCD. This fact, however, does not spoil the smoothness of the limit. Indeed, it simply means that the interaction terms, which are suppressed by inverse powers of $F$ as shown in eqs. (18) and (19), go to zero. The $U(1)_{R}$ symmetry is broken by an "infinite" amount and the low-energy theory consists of a single noninteracting chiral superfield. In the theory under discussion, the divergence associated to $F$ does not affect any physical quantity. We may ask what happens if we couple the $U(1)_{R}$ current to extra matter multiplets, analogously to the case of weak interactions, where some of the left-handed weak currents, coupled to ordinary matter, may coincide with the

\footnotetext{
${ }^{5}$ With the standard abuse of notation we denote with the same symbol the superfield and its complex lowest component.

${ }^{6} \mathrm{I}$ thank R. Casalbuoni for suggesting this point to me.
} 
chiral QCD currents. Even in this case the observable quantities will depend on $F$ only through combinations that remain finite in the massless limit. For instance, the decay rate of the Nambu-Goldstone boson into a pair of matter fields is proportional to $|F|^{2} m_{\xi^{0}}$, still vanishing in the $m \rightarrow 0$ limit. The effective Lagrangian considered here allows a small mass expansion around the massless limit. When $m \ll \Lambda$, we can easily take into account the small non-vanishing mass and the small interactions of the chiral supermultiplet, by keeping a convenient number of terms in the expansion of eqs. (18) and (19).

The general case can be discussed along similar lines. If we perform the massless limit, keeping a fixed non-vanishing ratio among the quark masses च, we obtain exactly $M^{2}$ massless supermultiplets, corresponding to the spontaneous breaking of $S U(M) \times S U(M) \times$ $U(1)_{B} \times U(1)_{R}$. The transition to the massless limit is smooth, and no observable quantity becomes singular. The formally infinite decay constants suppress the interaction terms and the theory experiences a free phase. When small masses are turned on, the theory has a small departure from the free phase, with interaction terms that can be systematically computed through an expansion in inverse powers of the decay constants.

The Nambu-Goldstone variables occur naturally also for $M=N$. The quantum constraint (8) satisfied by the meson superfields along the mesonic branch can be explicitly solved by:

$$
\mathcal{M}=\Lambda^{2} \exp \left(i \frac{\xi^{a} T^{a}}{\Lambda}\right),
$$

with $a=1, \ldots, N^{2}-1$. More precisely, the quantum constraint defines a complex nonlinear $\sigma$-model associated to the coset space $S L(N, C) \times S L(N, C) / S L(N, C)$. Indeed the constraint is invariant under independent $S L(N, C)$ transformations acting from the right and from the left on $\mathcal{M}$. Moreover, the generic vacuum configuration $\left\langle\mathcal{M}_{j}^{i}\right\rangle$ can always be mapped into $\Lambda^{2} \delta_{j}^{i}$ by means of a suitable $S L(N, C) \times S L(N, C)$ transformation, thus displaying the invariance of the vacuum under the diagonal $S L(N, C)$ subgroup. The generic coset element leads to the above representation for the meson variables [15]. It is interesting to note that in the chiral limit the decay constant is just $\Lambda$, which coincides with a naive extrapolation of the result (27) obtained by taking $M=N$, before performing the massless limit.

Although this physical picture is quite natural, we should be careful before drawing any conclusion about the massless limit for $M<N$. When the meson variables are used, the massless limit does not exist. The metric of the scalar manifold is singular for infinite meson VEVs. This singular behaviour is smoothed out by adopting the Nambu-Goldstone superfield $\xi^{a}$ and transferred to the decay constants of the theory. Nevertheless several questions remain open. Is there anything against using $\xi^{a}$ instead of $\mathcal{M}$ ? On what basis can we decide about the appropriate variables for the massless case? More generally: has the singular behaviour of mesons a physical meaning or does it simply reflect the inadequacy of the meson coordinates to describe the massless case? We have not yet found a sharp

\footnotetext{
${ }^{7}$ If some of the masses run to zero faster than the others, we can eliminate the "slow" modes by decoupling them from the effective theory.
} 
test that may discriminate between the two possibilities, and our present understanding only suggests that a smooth massless limit of SQCD, $M<N$, is not inconceivable.

\section{Conclusions}

In this work we have discussed in detail the properties of SQCD, $M<N$, for masses smaller than or comparable to the SQCD scale $\Lambda$. To start with, we have moved from meson variables to Nambu-Goldstone-like variables $\xi^{a}$. For small quark masses, the meson superfields experience very large fluctuations and a more convenient description of the system is obtained by using Nambu-Goldstone superfields, which fluctuate close to the origin in field space. We have presented explicit expressions for the mass spectrum and the decay constants of the system, checking that our results satisfy the properties required by decoupling. When the massless limit is approached, each observable varies smoothly, and the theory approaches a free phase, consisting of $M^{2}$ non-interacting chiral supermultiplets. If the massless limit exists, as supported by rephrasing the theory in terms of NambuGoldstone variables, the free phase has a clear physical meaning: it corresponds to the spontaneous breaking of the global flavour symmetry. The flavour symmetry is formally broken by an infinite amount, but this has the only effect of turning off the interactions between the chiral superfields. The massive case, for masses smaller than $\Lambda$, can be thought of as a perturbation around the massless case, with correction terms that can be systematically computed in the low-energy theory.

The discussion outlined in this paper makes use of an effective-Lagrangian approach. It is worth remembering that, besides the virtues and the relative handiness of the method, this approach has to face important limitations. These are related to the well-known fact that the correct identification of the elementary excitations of the system is part of the starting assumptions, not the outcome of a dynamical computation. Precisely for this reason, within this approach, we have not been able to resolve the ambiguity associated to the massless limit, and the picture presented here should be taken as a conjecture. It would be extremely interesting if some indications in favour or against our proposal could come from the intensively studied and rapidly evolving field of D-brane and Mtheory constructions where, already now, many non-perturbative results of super-YangMills systems have been reproduced [16].

\section{Acknowledgements}

I have enjoyed useful discussions with L. Alvarez-Gaumé, R. Casalbuoni, J. P. Hurni, C. Kounnas, E. Rabinovici, L. Randall, R. Rattazzi and F. Zwirner. A special thank goes to G. Veneziano for reading the manuscript and for several comments and suggestions. 


\section{References}

[1] For recent reviews see: K. Intriligator and N. Seiberg, Nucl. Phys. Proc. Suppl. B 45 (1996) 1 and Nucl. Phys. Proc. Suppl. B 55 (1996) 200;

M. Peskin, in the Proceedings of the 1996 TASI, Boulder, Colorado, USA, 1996 (hepth/9702094);

M. Shifman, lectures at the Summer School in High-Energy Physics and Cosmology, ICTP, Trieste, Italy, 1996 (hep-ph/9704114);

L. Alvarez-Gaumé and F. Zamora, preprint CERN-TH-97-257 (hep-th/9709180).

[2] T. Banks and E. Rabinovici, Nucl. Phys. B 160 (1979) 349;

E. Fradkin and S. Shenker, Phys. Rev. D 19 (1979) 3682;

S. Dimopoulos, S. Raby and L. Susskind, Nucl. Phys. B 173 (1980) 208.

[3] G. Veneziano, Phys. Lett. B 128 (1983) 199;

G. M. Shore and G. Veneziano, Int. J. of Mod. Phys. A 1 (1986) 499.

[4] T.R. Taylor, G. Veneziano and S. Yankielowicz, Nucl. Phys. B 218 (1983) 493;

G. Veneziano and S. Yankielowicz, Phys. Lett. B 113 (1982) 321.

[5] E. Witten, Nucl. Phys. B 202 (1982) 253.

[6] F. Buccella, J.P. Derendinger, S. Ferrara and C. Savoy, Phys. Lett. 115 B (1982) 375;

M. Luty and W. Taylor, Phys. Rev. D 53 (1996) 3399.

[7] I. Affleck, M. Dine and N. Seiberg, Nucl. Phys. B 241 (1984) 493.

[8] D. Amati, K. Konishi, Y. Meurice, G.C. Rossi and G. Veneziano, Phys. Rep. 162 (1988) 169, and references therein.

[9] N. Seiberg, Phys. Rev. D 49 (1994) 6857.

[10] A.C. Davis, M. Dine and N. Seiberg, Phys. Lett. B 125 (1983) 487.

[11] O. Aharony, M. Peskin, J. Sonnenschein and S. Yankielowicz, Phys. Rev. D 52 (1995) 6157

S.P. Martin and J. D. Wells, hep-th/9801157.

[12] M. T. Grisaru, M. Rocek and R. von Unge, Phys. Lett. B 383 (1996) 415.

[13] I. Affleck, M. Dine and N. Seiberg, Nucl. Phys. B 256 (1985) 557.

[14] E. Poppitz and L. Randall, Phys. Lett. B 336 (1994) 402.

[15] W. Lerche, Nucl. Phys. B 238 (1984) 582;

W. Buchmüller and W. Lerche, Ann. Phys. 175 (1987) 159;

G. M. Shore, Nucl. Phys. B320 (1989) 202;

A.C.W. Kotcheff and G. M. Shore, Int. J. Mod. Phys. A 4 (1989) 4391. 
[16] E. Witten, Nucl. Phys. B 507 (1997) 658;

A. Brandhuber, N. Itzhaki, V. Kaplunovsky, J. Sonnenshein and S. Yankielowicz, Phys. Lett. B 410 (1997) 27;

S. Elitzur, A. Giveon, D. Kutasov, E. Rabinovici and A. Schwimmer, Nucl. Phys. B 505 (1997) 202;

K. Hori, H. Ooguri, Y. Oz, preprint LBL-40336 (hep-th/9706082). 